\title{
DAGLI ARCHIVI DI MEDIOBANCA UN ISTITUTO DIVERSO
}

\author{
di Fulvio Coltorti
}

"So elusive a figure was Cuccia that those who knew him couldn't even figure out his age" 1

\section{L'archivio storico}

Nel novembre 2019 MEDIOBANCA ha aperto l'archivio storico alla consultazione pubblica arricchendo l'insieme dei materiali ora disponibili. Questo scritto ha lo scopo di riflettere sull'opportunità di rivedere alcuni giudizi espressi in passato sulla banca di Enrico Cuccia. Ho avuto l'incarico di istituire e organizzare questo archivio fino al 2015, opera nella quale mi sono avvalso della collaborazione di Giampietro Morreale (già direttore dell'archivio storico del Credito Italiano). Cominciai ad occuparmene all'inizio degli anni 2000 quando dirigevo l'Area Studi e Vincenzo Maranghi (allora amministratore delegato) mi chiese di riflettere su come organizzare le carte che avrebbero dovuto farne parte. Dopo la sua scomparsa nel luglio 2007 l'archivio storico gli fu intitolato (delibera del 27 giugno 2012). Nel giugno 2014 è stato dichiarato archivio di "interesse storico particolarmente importante" ai

Dipartimento di Storia dell'economia, della società e di Scienze del territorio "Mario Romani”, Università Cattolica del Sacro Cuore, Milano.

Francesco Cesarini, Gianpiero Fumi, Marco Onado e Marco Vitale hanno letto una versione precedente di questo scritto comunicandomi preziose osservazioni. Li ringrazio precisando che essi non hanno alcuna responsabilità sulla versione che qui si pubblica, la quale resta a mio esclusivo e personale carico. Le mie fonti sono costituite, oltre che dagli archivi storici aperti al pubblico, dalle molte riflessioni che ho avuto con Enrico Cuccia, Vincenzo Maranghi e Silvio Salteri nel corso del mio pluriennale lavoro in Mediobanca.

1 C. Reich, Financier. The Biography of André Meyer, New York, William Morrow and Company, 1983, p. 295. 
sensi delle leggi sui beni culturali, qualifica che lo ha posto sotto la tutela e la vigilanza della Soprintendenza archivistica lombarda. Il 31 gennaio 2013 è stato aperto un primo sito internet rendendo disponibili alcune carte fondamentali sulla storia di MEDIOBANCA, sui più importanti personaggi che l'hanno distinta insieme con le edizioni storiche delle pubblicazioni. Il progetto iniziale prevedeva l'accesso internet da remoto; ma nel 2019 l'apertura alla consultazione delle prime carte è avvenuta secondo le vecchie modalità che richiedono la presenza fisica dello studioso ${ }^{2}$.

MEDIOBANCA ha promosso alcuni approfondimenti storici dai quali hanno avuto origine sinora due pubblicazioni: la prima sulla "questione Olivetti" e la seconda sul profilo internazionale scelto sin dalle origini ${ }^{3}$. Sono documenti che si aggiungono a quelli già disponibili nel sito internet. Coprono aspetti sui quali gli studiosi non avevano potuto sviluppare riflessioni sufficientemente documentate, pervenendo il più delle volte ad interpretazioni parziali, non sempre giustificate dai fatti accaduti e influenzate da lavori di terzi che in molti casi hanno distorto la realtà. Occorre anche citare due libri, il primo di Giorgio La Malfa ${ }^{4}$ e il secondo di chi scrive, nei quali le "storie" di Enrico Cuccia e di MEDIOBANCA sono state raccontate con maggiore precisiones. In queste due opere gli autori hanno dato interpretazioni quali persone "informate dei fatti". Ė bene che il lettore sia edotto del rapporto personale che essi hanno avuto con MEDIOBANCA. Circa il merito della mia intervista lo storico Franco Amatori ha scritto un contributo (allegato al mio libro, p. 71) nel quale precisa che si "richiede assolutamente (fa parte del giuramento di Ippocrate di uno storico) una verifica". Ora queste "verifiche" sono possibili e lo saranno in misura sempre più ampia, man mano che si apriranno alla consultazione nuove carte.

2 Evitare agli studiosi viaggi inutili per accedere ai documenti mi sembrava, e mi sembra, una condizione essenziale nell'era dell'interconnessione e della globalità.

3 G. Morreale, Mediobanca e il salvataggio Olivetti. Verbali delle riunioni e documenti di lavoro 1964-1966, Milano, Mediobanca, 2019; G. FARESE, Mediobanca e le relazioni economiche internazionali dell'Italia. Atlantismo, Integrazione europea e sviluppo dell'Africa, 1944-1971, Milano, Mediobanca, 2020.

4 Responsabile scientifico dell'Archivio storico.

5 G. La Malfa, Cuccia e il segreto di Mediobanca, Milano, Feltrinelli, 2014; F. Coltorti (a cura e con G. Giovannetti), La Mediobanca di Cuccia, Torino, Giappichelli, 2017. 


\section{La riservatezza}

MEDIOBANCA è stata costituita il 10 aprile 1946 dalle tre banche allora qualificate "di interesse nazionale" (COMIT - Banca Commerciale Italiana, CREDIT - Credito Italiano e Banco di Roma). Occorreva recuperare l'attività delle banche d'affari (banche miste), letteralmente cancellate dalla riforma del 1936. Era stata la reazione alla nostra maggiore crisi finanziaria che le aveva viste in stato fallimentare richiedendo nel 1933 il loro salvataggio da parte dello Stato. La riforma aveva disposto la specializzazione separando il credito ordinario (a breve termine) dal credito mobiliare (a medio e lungo termine). Le banche salvate, che avevano scelto il breve termine, si erano anche impegnate "moralmente" ad evitare il ritorno alla presa di partecipazioni industriali6. La storia di MEDIOBANCA è quindi breve, ma nonostante ciò ancora poco conosciuta. Finora, coloro che l'hanno esaminata hanno dovuto fare i conti da un lato con il successo indubbio, che non poteva non richiamare critiche strumentali dai concorrenti, e, dall'altro, con l'estrema riservatezza del fondatore. Enrico Cuccia non rilasciò mai interviste e condusse un'esistenza lunga e operosa (92 anni, 54 dei quali trascorsi nel suo ufficio milanese in via Filodrammatici 10). Una vita, la sua, scevra di protagonismo e molto riservata similmente a quella del principale mentore, Donato Menichella.

Tutta questa riservatezza non ha impedito di conservare accuratamente la documentazione dell'attività, il che rende ancor più interessante l'archivio 7 . MEDIOBANCA non ha archiviato solo le operazioni proprie, ma ha contribuito in misura non lieve alla trasparenza del mercato finanziario attraverso le modalità dei collocamenti (introducendo, senza obblighi normativi, i prospetti illustrativi degli emittenti secondo

6 Per maggiori dettagli si veda F. COLTORTI, Banche e Borsa Valori nell'Italia in transizione, in "Il Politico" n. 3, 2017, p. 224 sgg..

7 Le relazioni annuali sono state raccolte e pubblicate nel 2007. Si veda E. CuCCIA, Relazioni di bilancio Mediobanca 1947-1982, Milano, Mediobanca, 2007. Il banchiere ha rivestito la carica di Direttore Generale dal $1^{\circ}$ giugno 1946 al 29 settembre 1982 quando gli subentrò Silvio Salteri. Rimase poi membro del Consiglio di amministrazione fino al 1988. Con i “cucciani” Silvio Salteri (1982-1988) e Vincenzo Maranghi (1988-2003) amministratori delegati partecipò sempre in qualità di invitato alle sedute del Consiglio di amministrazione. Questa partecipazione era stata richiesta dal Consiglio il 14 marzo 1988 in occasione dell'acclamazione a Presidente d'onore. 
gli usi dei mercati internazionali), le pratiche di finanziamento (con il monitoraggio continuo dei clienti) e promuovendo pubblicazioni periodiche dell'Area Studi. Queste hanno reso visibili le stesse operazioni della banca, anche se necessariamente ex post. Le opere di cui si parla comprendono raccolte di indici su titoli quotati, dati di borsa ed elementi vari di società quotate e non quotate, nonché elaborazioni di schede monografiche sulle principali società e gruppi italiani ${ }^{8}$. L'Area Studi ha inoltre pubblicato indagini settoriali e predisposto tecniche statistiche con sostanziali innovazioni nel panorama delle banche d'investimento italiane ed estere: dati di bilancio cumulativi delle principali società italiane (iniziati negli anni ' 60 del secolo scorso), aggregati dei bilanci delle maggiori multinazionali (iniziati nel 1995) e delle maggiori banche internazionali (dal 2002), indicatori del mercato azionario e approfondimenti sull'organizzazione delle borse valori. Questi materiali, promossi da quella che chiamiamo la MEDIOBANCA di Cuccia ${ }^{9}$, sono attualmente disponibili nel sito internet di cui s'è detto sopra.

\section{Giudizi da rivedere}

Fermo il generale riconoscimento di un'elevata competenza tecnica, le opere di Enrico Cuccia e del suo istituto sono state oggetto di ammirazione come pure di disapprovazione e di critiche assai dure. Le principali critiche sono venute da tre generi di osservatori: politici, storici e pubblicisti. Possiamo distinguere due diversi periodi. L'attività iniziale venne rivolta a "costruire" una banca seria e competente e gli anni '40-'60 videro il sostegno alla ricostruzione dell'apparato industriale italiano dotandolo di moderni mezzi produttivi e organizzativi. Fino agli anni del miracolo economico (concluso nel 1963), l'immagine dell'istituto non fu in discussione. La migliore sintesi di questi giudizi sta nella lettera che, su ispirazione di Raffaele Mattioli, i membri del Consiglio di amministrazione e del Collegio sindacale spedirono a Enrico Cuccia il 28 ottobre 1966, nella ricorrenza dei 20 anni dalla fon-

8 Tra cui gli Annuari R\&S, pubblicati dal 1976 al 2019, costituiti da 82 volumoni per complessive 117 mila pagine.

9 Ovvero la Mediobanca che va dalla costituzione all'allontanamento di Vincenzo Maranghi, ultimo "cucciano", nel 2003. Per maggiori dettagli rimando al mio libro, già citato. 
dazione. Tra l'altro vi si scriveva: “... non vogliamo lasciar passare il quadrilustre anniversario della fondazione di MEDIOBANCA senza esprimerLe, a Lei come primo artefice delle sue fortune, il nostro compiacimento e il nostro ringraziamento per il lavoro compiuto con così paziente fervore, tranquillo entusiasmo e sempre vigile intelligenza" 10 . Il tono di questa lettera non riflette solo una banale ricorrenza, ma dimostra che la fase di impostazione di MEDIOBANCA era pienamente condivisa, soprattutto da Mattioli. Alcuni lo hanno messo in dubbio soprattutto prendendo lo spunto da una bozza di missiva del 1961 nella quale il banchiere della COMIT chiedeva "nell'interesse di chi è ammnistrata MEDIOBANCA?" Questa lettera fu citata da Giorgio Rodano che le diede il senso del disappunto di Mattioli per un eccessivo attivismo nel campo del merchant banking. Sulla vicenda specifica non sappiamo molto salvo che il vero oggetto del contendere fosse l'autonomia di MEDIOBANCA nel decidere i rischi da assumere indipendentemente dagli interessi dei soci ${ }^{11}$. Già nel 1957 vi era stata una frizione a proposito della politica delle banche di credito ordinario di mascherare operazioni finanziarie con prestiti formalmente a breve scadenza, ma di fatto soggetti a rinnovo. I grandi gruppi traevano beneficio dai più convenienti tassi a breve ed evitavano quindi di rivolgersi a MEDIOBANCA. In quell'occasione Mattioli espresse il desiderio che MEDIOBANCA rilevasse dalle BIN quei rischi "anomali" dal punto di vista formale. Circa l'esito di questo confronto, i colleghi mi hanno riferito che gli affari trasferiti dalle BIN continuarono ad essere guardati con occhio assai "vigile"12.

10 Vorrei anche citare il giudizio fattomi avere nel settembre 2017 da Tancredi Bianchi (un grande studioso e un'importante personalità nel mondo bancario): “un banchiere eccezionale e un uomo di grandi valori intellettuali e morali. Con il gusto, quasi artistico, di sapere proporre al 'cliente', ossia nella contingenza al conferente un mandato, la soluzione corretta per attuare scelte finanziarie di azienda, che si sarebbero riflesse in un ritrovato equilibrio economico di gestione, comunicando nel contempo al mercato che quelle opzioni erano, insieme, la soluzione più elegante".

${ }^{11} \mathrm{Si}$ veda G. La MALfa, Cuccia e il segreto di Mediobanca, cit., pp.188-189. Il libro di G. Rodano, Il Credito all'economia. Raffaele Mattioli alla Banca Commerciale Italiana (Milano-Napoli, Ricciardi editore, 1983), aveva preso lo spunto dallo stesso Mattioli (p. IX). La lettera, citata da Rodano a p. 167, era precedente al "panegirico" per il quadrilustre. In Mediobanca si riteneva che fosse stata una reazione al rifiuto di aiutare la casa editrice Giulio Einaudi, che versava in cattive acque.

12 S. GerBI, Mattioli e Cuccia. Due banchieri del Novecento, Torino, Einaudi, 2011, pp. 142-151. Lo stesso Gerbi dà conto dell'atteggiamento di Cuccia verso la casa editrice 
Dopo il miracolo economico il contesto mutò. Negli anni '70 le grandi imprese italiane finirono in una crisi molto grave che le destabilizzò richiedendo operazioni straordinarie per il loro salvataggio. Le consulenze per fusioni e ristrutturazioni si aggiunsero ai finanziamenti per lo sviluppo che erano stati il mestiere prevalente agli esordi. La competenza maturata da MEDIOBANCA le consentì di assorbire le operazioni richieste dalle imprese più importanti e questo spostò l'attenzione dei critici dalla competenza tecnica all'esercizio del "potere" che derivava dal suo uso. In particolare di politici, studiosi e concorrenti, nonché di molti pubblicisti i quali si erano trovati preclusi alcuni dei principali strumenti del mestiere, le interviste. Ricordo che un bravo giornalista, Fabio Tamburini, nel 1992 scrisse un libro su Enrico Cuccia sollecitato, come lui stesso riferì, da un "mandante" suo amico, Cesare Merzagora ${ }^{13}$. Nella iniziale nota dell'autore si legge: "L'esile Cuccia, vestito di scuro con l'inseparabile cravatta nera, piccolo di statura, all'apparenza fragile ma con uno sguardo penetrante, refrattario a qualsiasi forma di protagonismo, ha conquistato tutto il suo potere grazie a capacità professionali indiscusse..." (pp. 7-8). Ma nel retro della copertina si dava spazio ad un ignoto concorrente: "uno dei finanzieri più brillanti tra quelli che operano sulla piazza di Milano descrive il ruolo svolto da Cuccia equiparandolo a una sorta di Robin Hood della finanza. Naturalmente alla rovescia e cioè 'impegnato a sottrarre risorse ai risparmiatori e al mercato per aumentare il tesoro dei ricchi' ". Cuccia non ha mai voluto saperne di quel libro. Aveva declinato un anno prima la richiesta dell'autore di intervistarlo e quando gli fece omaggio di una copia del libro rispose ringraziando, ma precisando "non lo leggo". Una decina di giorni dopo ricevette da Natalino Irti il testo del suo intervento alla presentazione pubblica del libro, ma, di nuovo, nel ringraziare precisò di non avere nessuna intenzione di leggerlo, negando per giunta la sua "sicilianità" (infatti era nato a Roma e viveva a Milano).

La riservatezza con la quale Enrico Cuccia ha sempre condotto gli affari è stata una critica diffusa proveniente dai pubblicisti. Ma va richiamata l'etica del banchiere per il quale non può esistere alcuna fiducia da parte dei clienti in assenza di riservatezza sulla conduzione e l'assistenza

che era ditta unipersonale: “...una trasformazione giuridica dell'azienda metterebbe ancora più in evidenza il problema del rapporto tra i mezzi propri e i mezzi dei terzi, sottolineando la necessità di procedere in primo luogo ad una sistemazione del capitale", p. 152.

${ }^{13}$ F. TAMBURINI, Un siciliano a Milano, Milano, Longanesi \& C. editore, 1992. 
ai loro affari. In più, incideva non poco lo stile di vita lontano dagli eventi mondani, anche qui sull'esempio di Menichella. Questi principi non hanno limitato la collaborazione con le istituzioni. Enrico Cuccia ha partecipato a due audizioni in Senato, il 12 febbraio 1975 sul funzionamento della Borsa valori in Italia e il 30 maggio 1978 sul finanziamento delle imprese industriali in Italia. Ha promosso lavori innovativi sulla finanza pubblica in Italia, sulla politica dei redditi, sull'edilizia popolare, consegnato relazioni utili alle privatizzazioni e all'ingresso nell' età dell'euro ${ }^{14}$, ideato indicatori utili al Servizio sanitario nazionale ${ }^{15}$. Nel 1986 promosse un progetto di istituto privato per la cura e la ricerca oncologica che si concretizzò nel 1994 nella fondazione dello IEO Istituto Europeo di Oncologia, affidato a Umberto Veronesi.

\section{Il monopolista}

Una seconda, diffusa critica riguarda la posizione monopolistica assunta da MEDIOBANCA. Al momento della sua nascita il settore creditizio era formato da enti pubblici e da banche sotto la forma giuridica di società per azioni gestite secondo criteri privatistici. Il credito a media e lunga scadenza riguardava molti istituti tra i quali alcuni dotati di consistenti patrimoni, come l'IMI (Istituto Mobiliare Italiano), l'ICIPU (Istituto di Credito per le Imprese di Pubblica Utilità), il CREDIOP (Consorzio di Credito per le Opere Pubbliche) e le sezioni speciali di alcuni enti pubblici bancari e casse di risparmio con attività prevalente di credito ordinario (tra loro: Banca Nazionale del Lavoro, Istituto Bancario San Paolo di Torino, Monte dei Paschi di Siena, Banco di Napoli, CARIPLO). Vi erano poi i mediocrediti regionali, enti pubblici orientati principalmente - ma non esclusivamente - verso le piccole imprese; il principale, quello Lombardo, fu costituito nel 1953 per iniziativa della CARIPLO con l'apporto di 118 enti creditizi e assicurativi ${ }^{16}$.

14 Tra cui i quaderni pubblicati nel 1996 e 1997 dall'Istituto Ugo La Malfa che contribuì a scrivere.

15 Servizio gratuito al Ministero della Sanità attraverso i lavori di un Gruppo di clinici di grande prestigio e di consulenti coordinati dall'Ufficio Studi (1993 e 1997). Compresa una sperimentazione degli indicatori in un gruppo di ospedali.

${ }^{16} \mathrm{Al} 31$ dicembre 1979 gli azionisti principali erano la CARIPLO con il 62,01\% del 
Inoltre, con MEDIOBANCA nacquero e si svilupparono altri istituti a lei simili, in quanto emanazioni di aziende di credito ordinario gestite privatisticamente e aventi la forma giuridica di società di capitali: CENTROBANCA (costituita nello stesso 1946 dalle banche popolari), EFIBANCA (nata come ente finanziario e riorganizzata come istituto a medio termine nel 194917) e INTERBANCA (costituita nel 1961 con azionariato privato ${ }^{18}$ ). La provvista di questi enti era costituita da depositi vincolati e obbligazioni. Gli enti pubblici erano ovviamente garantiti dallo Stato che sosteneva le obbligazioni che emettevano e i depositi che assumevano. Nel comparto del credito mobiliare MEDIOBANCA è stata pertanto uno dei molti operatori, uno dei più recenti e sicuramente non il più potente in termini di mezzi finanziari disponibili.

Alcune aree del mondo politico hanno criticato MEDIOBANCA accusandola di avversione al mercato auspicandone la "liberazione" per (ri?) stabilire la "democrazia economica". Principalmente si è trattato di Beniamino Andreatta e degli studiosi a lui vicini. Ritengo che queste posizioni fossero il frutto di intenzioni politiche e non personali. Il pensiero di Andreatta lo si può desumere da una pubblicazione del 198419 dove richiama esplicitamente la presunta posizione monopolistica, l'operare a supporto dei gruppi di controllo esistenti ("visione elitistica e ristretta") e l'eccessiva dimensione delle operazioni. Auspicava inoltre la costituzione di altre banche d'investimento, meglio se non promanassero dal mondo bancario.

Ritengo legittime, ma deboli queste argomentazioni. Più probabile che vadano viste in quello spirito di Reconquista del potere bancario da parte del partito di maggioranza di cui ha parlato Giancarlo Galli, giornalista molto vicino all'economista e politico democristiano ${ }^{20}$. In

capitale, le tre BIN con il 10,75\% e altri 90 enti con il restante $27,24 \%$ (fonte: $R \& S 1979$, p. 563 del primo volume).

${ }^{17}$ Fondata nel 1939 da IRI e altre principali società industriali. Nel 1977 i principali azionisti erano Banca Nazionale del Lavoro, Italmobiliare (Pesenti), Banco di Sicilia, Monte dei Paschi di Siena, San Paolo di Torino, Banco di Napoli e Banca Popolare di Novara (fonte $R \& S$ 1977). Nel 1984 i soci di controllo divennero Banca Nazionale del Lavoro e Banca Popolare di Novara (paritetici al 30,53\%).

18 Principali soci: Banca Nazionale dell'Agricoltura, Banco Ambrosiano, Banca d'America e d'Italia e Istituto Centrale di Banche e Banchieri.

${ }^{19}$ B. AndreatTa, Le poche cose da fare in F. A. Grassini (a cura), "Le banche e il capitale di rischio: speranze o illusioni?", Bologna, il Mulino, 1984, p. 207 sgg..

${ }^{20}$ G. Galdi, Nella giungla degli gnomi. Milano, Garzanti, 2008, p. 99. Citando 
primo luogo perché MEDIOBANCA non è mai stata monopolista per suo volere. Fin dalla nascita, come visto, è stata tra una moltitudine di istituti ben più potenti. Non ha mai avuto un'esclusiva da sfruttare, requisito essenziale di ogni monopolio. La sua posizione preminente è sempre stata il frutto della sua competenza e questa è stata accumulata grazie all'ingegnosità, alla moralità e alla correttezza negli affari del suo fondatore e dei collaboratori di cui si è circondato. Ha beneficiato del rapporto con le banche fondatrici, ma non è vero che abbia operato traendo diretto vantaggio dalla finanza pubblica. Le tre BIN (controllate dall'IRI) fornirono il patrimonio iniziale e, dietro il pagamento di commissioni di sportello, l'infrastruttura al dettaglio per la raccolta; ma obbligazioni e depositi vincolati furono sempre collocati alle condizioni di mercato senza alcuna garanzia pubblica. Garanzia che fu deliberatamente esclusa al momento della fondazione: "nelle operazioni concluse dalla MEDIOBANCA non è concessa la garanzia dello Stato e non è previsto alcun contributo del Tesoro al pagamento degli interessi dovuti dai mutuatari $[\ldots]$ l'istituto sarà indotto ad essere maggiormente guardingo nell'esame tecnico dei prestiti da concedere e potrà più facilmente resistere alle pressioni di natura politica che fatalmente intralciano l'attività delle banche" 21 .

La visione "elitistica" non è infondata. Richiama la formazione di un "circolo" di imprese che molti battezzarono "salotto buono". Le relazioni più strette riguardavano Fiat, Pirelli, Montedison, Assicurazioni Generali, Fondiaria, Snia Viscosa, Olivetti22. Questo circolo fu parte della strategia cucciana secondo la quale una banca d'affari che voglia

Andreatta da "cristiano adulto" descrive le sue azioni: "lavora per un preciso disegno, prendendo a prestito il comportamento dei liberali, nell'immediato dopoguerra... erano riusciti a conservare gran parte del potere reale installandosi nei gangli della finanza, sfruttando i rapporti internazionali. 'Lo hanno potuto fare, avendo una classe dirigente', martellava. Indicando in Cuccia-Mediobanca il punto di riferimento di questa galassia. Fra i primi gesti impone la nomina di Romano Prodi alla presidenza dell'IRI. L'istituto che azionariamente controlla (senza però mai riuscire ad esercitare le sue prerogative) la stessa Mediobanca". Passa poi a ricordare il sostegno a Giovanni Bazoli che in effetti riuscirà con l'Intesa San Paolo [...] "nella legittima ambizione di trasformarsi $[\ldots]$ in un 'nuovo Cuccia' "(pp.98-99).

${ }^{21}$ Così Giordano Dell'Amore in un articolo su Il Popolo del 3 ottobre 1946, riprodotto in F. Coltorti, La Mediobanca di Cuccia, Torino, Giappichelli 2017, pp. 111-112.

${ }^{22} \mathrm{Si}$ veda l'edizione 1978 dell'Annuario $R \& S$ (scheda Mediobanca, principali partecipazioni minoritarie; pp. 1532-1533. 
essere attiva deve restare a stretto contatto con gli ambienti nei quali gli affari si formano. È stato così per le relazioni internazionali nel momento della fondazione, quando la banca venne pensata come ente al servizio dell'economia di un Paese povero di risorse materiali e finanziarie, ma ricco di uomini desiderosi di riscatto ${ }^{23}$. È stato così anche per l'ingresso nei preesistenti patti di sindacato delle grandi imprese (tra i primi, Bastogi, Montecatini, Pirelli, Fondiaria). Il "circolo" di MEDIOBANCA può essere visto in negativo pensando agli imprenditori privati che ne facevano parte, ma che operavano liberamente anche con altre banche. Essi, alla lunga, sono divenuti, anche per il proprio tornaconto, i veri responsabili della sua natura elitaria; ma non era un circolo chiuso. Intanto perché includeva le molte imprese del Gruppo IRI; e poi perché occorreva poter lavorare con tutti, possibilmente selezionando i clienti buoni e scartando i cattivi. È da questa selezione che si deve giudicare l'esito delle operazioni di MEDIOBANCA. A mio parere, alla domanda di Franco Amatori, se la banca di Cuccia abbia influito e in che modo sullo sviluppo economico italiano, va risposto che il segno più attendibile del suo contributo sta nell'esito degli impieghi. Qui l'istituto ha brillato di luce unica: lo $0,001 \%$ appena dei crediti è andato perduto; profetiche furono al riguardo le considerazioni di Giordano Dell'Amore citate sopra. Quanto poi alla sintesi della gestione, escludendo il 1947 (esercizio nel quale si decise di spesare tutti i costi di impianto), i bilanci furono chiusi sempre in utile mentre il valore di Borsa dell'azione, fatto 100 il 1956 (anno di prima quotazione) chiuse il settembre 1982 (anno delle dimissioni di Cuccia da direttore generale) a quota $6.758,9$ contro un livello dell'indice generale di mercato pari a 110,424. Questi risultati dimostrano che le operazioni fatte hanno avuto esito positivo e quindi hanno sostenuto lo sviluppo del Paese. Va aggiunto che MEDIOBANCA non si interessò mai direttamente della gestione industriale dei suoi clienti (una massima di Menichella) anche se ne era corresponsabile quando partecipava ai patti di sindacato. E difatti, l'aspetto più difficile della gestione era l'individuazione degli imprenditori e dei capitalisti adeguati alla bisogna a costo di "pesare" e non contare le loro azioni. In ogni caso, MEDIOBANCA è riuscita a te-

23 G. FARESE, Mediobanca e le relazioni economiche internazionali dell'Italia. Atlantismo, integrazione europea e sviluppo dell'Africa, 1944-1971, cit. p. 9.

${ }^{24}$ E. CucCIA, Relazioni di bilancio Mediobanca 1947-1982, cit., p. 385. 
nere in piedi e nel territorio nazionale le maggiori imprese private. Nel dopo Cuccia, invece, queste sono decadute rimpicciolendosi, delocalizzando, emigrando o finendo in mani straniere 25 .

In realtà, il mercato è sempre stato aperto e contendibile ed Enrico Cuccia, sin dall'inizio, ha tenuto presenti gli avvertimenti di Menichella sull'inframmettenza dei politici e sulla necessità di essere indipendenti nella gestione. Cuccia scriverà: "in tempi successivi alla caduta del fascismo si è determinata una situazione di condizionamenti politici in ampie aree di gestione della cosa pubblica, rendendo ancor più irripetibile l'esperienza legata al nome di Donato Menichella: egli è stato infatti [....] la persona che ha mantenuto viva una grande tradizione di indipendenza e di probità" 26 . Di ciò si rinviene traccia evidente nelle carte archiviate e nel lavoro di Giovanni Farese; ma occorre dire che il principio era anche nelle corde del principale critico, Beniamino Andreatta ${ }^{27}$.

\section{Capitalismo familiare e mercato}

La critica più insistente viene tuttavia da chi ha visto le operazioni di salvataggio delle imprese finalizzate al sostegno delle famiglie e contrarie all'obiettivo di sviluppare un mercato dei capitali più adeguato alle esigenze del Paese. "Soltanto in Italia [.... la maggior parte delle imprese private partecipa ad un unico e consolidato sistema di alleanze (di cui il gruppo Agnelli e MEDIOBANCA sono i membri dominanti), fatto che evidentemente non favorisce la competizione e il pluralismo"28. Ma, che cos'altro fare in un Paese nel quale l'economia è dominata da aziende familiari e da capitalisti che storicamente pre-

25 Rinvio alla relazione che tenni in Senato il 22 maggio 2017 al convegno Lo Stato azionista, finalità regole strumenti, pubblicata su "Bancaria", n. 9, 2017.

${ }^{26}$ E. CuCcia, Un esempio inimitabile, in "Donato Menichella. Testimonianze e studi raccolti dalla Banca d'Italia", Roma-Bari, Laterza, 1986.

${ }^{27}$ N. Andreatta, Per un'Italia moderna. Questioni di politica e di economia, Bologna, il Mulino, 2002: "il punto fondamentale [della legge bancaria italiana] rimane questa capacità di giudizio indipendente del banchiere" p. 152. Similmente in F. SALSANO, Andreatta Ministro del Tesoro, Bologna, il Mulino, 2009, pp. 96-97.

${ }^{28}$ F. AMATORI, F.Brioschi, Le grandi imprese private: famiglie e coalizioni, in F. BARCA (a cura), "Storia del capitalismo italiano dal dopoguerra a oggi", Roma, Donzelli, 1997 ; p. 149. 
feriscono la finanza all'industria? Con un mercato che non esisteva e che è stato svantaggiato da politiche errate? Dalla nazionalizzazione elettrica alle agevolazioni per lo sviluppo del Mezzogiorno ad ogni genere di aiuto ai grandi gruppi, forti del controllo dei media offerti ai politici che hanno predisposto quelle politiche?

Le relazioni più importanti sono state con due entità molto diverse tra loro che costituivano le maggiori concentrazioni capitalistiche del Paese: la Fiat controllata dalla famiglia Agnelli e la Montedison posseduta da un azionariato diffuso, discendente dallo stato di public company delle due aziende originali, Montecatini e Edison. Ha influito forse la scarsa importanza attribuita dagli studiosi alle strutture di comando dei capitalismi esteri con i quali si era in competizione.

Oltre a richiamare la battuta sulla Einaudi citata in precedenza, vale la pena di riflettere sulla prima vera operazione di banca d'affari: il salvataggio dell'Olivetti, un'impresa modello per la capacità innovativa unita alla bellezza dei prodotti e all'impegno sociale. Una storia ancora non del tutto capita. Tutto iniziò nel gennaio 1964 quando la famiglia Olivetti, preoccupata per le perdite emergenti, la salita dei debiti e l'assenza di manager capaci di gestire l'impresa dopo la scomparsa di Adriano nel 1960, incaricò Bruno Visentini di cercare un gruppo di salvatori ${ }^{29}$. Chiesero a Raffaele Mattioli il quale suggerì di rivolgersi a MEDIOBANCA. I problemi da risolvere erano sostanzialmente quattro: 1) trovare soldi per ricomperare le azioni che la famiglia aveva ceduto alle banche (prevalentemente estere) a garanzia di prestiti; 2) finanziare l'impresa che aveva subito e subiva deflussi di cassa per la cattiva gestione; 3) trovare un partner tecnico in grado di gestire le attività elettroniche dopo la scomparsa nel 1961 del direttore Mario Tchou; 4) ricapitalizzare. Il "Gruppo di intervento" fu costituito con IMI, La Centrale, Pirelli, Fiat che affiancarono MEDIOBANCA e la famiglia in un patto di sindacato dove questa aveva posto la condizione di essere in maggioranza. Il salvataggio implicò la cessione alla General Electric della Divisione elettronica, ossia della produzione dei grandi calcolatori. L'effetto di questa cessione è stato esagerato da una vulgata che lo ha segnato come grave colpo alla capacità dell'Italia di partecipare ad un

\footnotetext{
${ }^{29} \mathrm{Si}$ veda G. Morreale, Mediobanca e il salvataggio Olivetti. Verbali delle riunioni e documenti di lavoro 1964-1966, cit..
} 
settore fondamentale per lo sviluppo e di mantenere un'industria elettronica tout-court. Era possibile una soluzione diversa? Occorre considerare che la Fiat, comandata da Vittorio Valletta, aveva posto quella cessione come condizione essenziale alla sua partecipazione. Senza la Fiat il salvataggio non avrebbe potuto avere luogo. Inoltre, se pure le produzioni dell'Olivetti erano tecnicamente equiparabili a quelle delle grandi imprese americane, è anche vero che nel 1964 l'IBM, il produttore dominante con il $76 \%$ del mercato mondiale, lanciò la sua Serie 360 le cui innovazioni tecnologiche resero obsoleti gli Elea dell'Olivetti. Questo è il giudizio dato nel 2009 da Rosario Amodeo, Amministratore delegato della Engineering - Ingegneria Informatica ${ }^{30}$. La sopravvivenza dell'azienda italiana sarebbe quindi stata difficile e ciò è confermato dal fatto che la stessa General Electric, che aveva acquisito le tecniche Olivetti, dovette capitolare cedendo nel 1970 tutte le sue attività alla Honeywell. Ma vi sono due aspetti legati a questa cessione che sono stati a lungo strumentalizzati nella critica a MEDIOBANCA. Il primo riguarda il precursore del personal computer e cioè il modello P101 dell'Olivetti. Ebbene, quel progetto non venne ceduto, ma rimase in casa Olivetti; la quale non seppe dunque svilupparlo adeguatamente. Il secondo aspetto taciuto riguarda la stessa divisione elettronica che ai fini della cessione venne scorporata e conferita ad una società, Olivetti General Electric, inizialmente a partecipazione congiunta. Questa società nel 1970 mutò denominazione in Honeywell Information Systems Italia e conservò per lungo tempo la sua attività di produzione di calcolatori elettronici in Italia ${ }^{31}$.

Nel caso Fiat, MEDIOBANCA ha sempre sostenuto l'impresa fornendo i servizi finanziari che richiedeva. Quanto alla Montedison le operazioni hanno sempre puntato a tenere la società sul mercato borsistico sotto controllo privato. Le operazioni potevano essere congegnate in modo diverso? Sì, ma non v'è possibilità di valutare un effetto alternativo.

Val la pena ricordare il giudizio di due economisti che hanno messo a confronto l'attività degli 8 principali istituti italiani di credito mobiliare: "Alla luce dell'analisi svolta, l'affermazione di Andreatta [su ME-

${ }^{30}$ R. AMODEO, Olivetti nella grande sfida internazionale dell'informatica, in AAVV, "Olivetti, cento anni. Note di lettura", Roma, Engineering, 2009.

${ }^{31}$ Fabbrica a Caluso e laboratori di ricerca e progettazione a Pregnana Milanese. Si veda la voce Honeywell negli Annuari R\&S. 
DIOBANCA $N d A$ ] pare ingenerosa. Gli sviluppi che l'hanno guidata [...] paiono aver corrisposto a precise esigenze di garanzia degli equilibri di controllo all'interno del gruppo principale del capitalismo italiano; e, inoltre, vi erano da garantire anche gli equilibri tra questo gruppo e lo Stato, affare di cui MEDIOBANCA si faceva demiurgo, anche nelle proprie sembianze di centauro tra pubblico e privato. Non è chiaro come tali equilibri avrebbero potuto essere altrimenti salvaguardati”. Gli stessi hanno anche documentato il ruolo di MEDIOBANCA quale principale lead manager nelle IPO a valore negli anni 1981-19932.

Quanto infine all'accusa, pure frequente, di sopprimere la "democrazia economica" con l'uso dei patti di sindacato nella stabilizzazione degli assetti di controllo delle maggiori imprese, ancora nel 2018, quindici anni dopo la "disattivazione" della MEDIOBANCA di Cuccia, il $77 \%$ delle società quotate in Borsa a valore era soggetto al dominio di soci di controllo nelle sue varie forme (patti inclusi), contro il $61 \%$ nel 1998 (dati Consob). D'altro canto, se la democrazia dovesse essere giudicata dall'instabilità "creativa" generata dalla competizione di mercato si dovrebbero ricordare le grandi OPA ostili: la prima fu quella di Michele Sindona nel 1971 sulla Bastogi, la seconda quella della Fiat sulla Montedison nel 2001 e la terza quella dell'Olivetti di Colaninno sulla Telecom nel 1999. La prima non ebbe seguito; la seconda con la Fiat spalleggiata dalle maggiori banche (inclusa quella centrale), produsse come risultato: a) la conquista della Montedison da parte della Fiat e dell'alleato francese EDF; b) il suo smembramento con cessione delle attività elettriche ai francesi; c) l'assenza di vantaggi durevoli da tale smembramento a fronte di una caduta dello scalatore italiano in stato pre-fallimentare; d) la sparizione del secondo grande gruppo privato. L'esito della terza lo vediamo ancora nell'arretratezza della copertura digitale del nostro Paese. Difficile pensare che pluralismo e democrazia si perseguano in tale maniera; sono certamente determinanti le persone che attivano queste operazioni come pure gli enti finanziari che le sostengono.

MEDIOBANCA avrebbe potuto indirizzare diversamente le azioni dei grandi gruppi italiani inducendoli ad operazioni sul mercato invece che

${ }^{32}$ M. De CeCCO, G. FerRI, Le banche d'affari in Italia; Bologna, il Mulino, 1996, pp. 106-108 e Tab. 14. 
al di fuori di esso? Finita la MEDIOBANCA di Cuccia e rimosso per consunzione il salotto buono, i nostri grandi imprenditori paiono ora ridotti ai "capitani di sventura" che attendono ancora di essere realmente privatizzati ${ }^{33}$. Il giudizio finale su MEDIOBANCA resta aperto.

“Chiste so' i sunaturi e cu chiste s'adda sunà!" (Donato Menichella ${ }^{34}$ )

\begin{abstract}
This article takes its cue from the opening to the public of the MEDIOBANCA Historical Archive and, based on the many documents now available, proposes to reconsider some criticisms addressed in the past to the bank. The allegations of monopoly and anti-competitive transactions in a financial
\end{abstract}

market which was free and contestable seem unfounded. The judgment on the so-called "good drawing room" remains open. But it must be reconsidered in the light of the operations of the major private groups, before and after Enrico Cuccia whose figure continues to be evoked.

${ }^{33}$ M. Borsa, L. De Biase, Capitani di sventura. Agnelli, De Benedetti, Romiti, Ferruzzi, Gardini, Pirelli: perché rischiano di farci perdere la sfida degli anni '90; Milano, Mondadori, 1992.

${ }^{34}$ Ripresa da V. Menichella, Donato Menichella. Un silenzioso e sconosciuto uomo del Sud, Banca Popolare Sud Puglia, Galatina, 1986, p. 46. 